\title{
La multiplicación de las penas: cuestiones preliminares para el abordaje de acusaciones y denuncias de proximidad en tiempos de pandemia*
}

\author{
María E. Epele ${ }^{* *}$ \\ Consejo Nacional de Investigaciones Científicas y Técnicas (Conicet), \\ Universidad de Buenos Aires, Argentina
}

\section{https://doi.org/10.7440/antipoda44.2021.06}

Cómo citar este artículo: Epele, María E. 2021. "La multiplicación de las penas: cuestiones preliminares para el abordaje de acusaciones y denuncias de proximidad en tiempos de pandemia". Antípoda. Revista de Antropología y Arqueología 44: 119-144. https://doi.org/10.7440/ antipoda44.2021.06

Recibido: 15 de noviembre de 2020; aceptado: 29 de abril de 2021; modificado: 25 de mayo de 2021.

Resumen: con la irrupción de la pandemia por el SARS-CoV-2 y su devastador avance se implementaron diferentes formas de confinamiento que en Latinoamérica transformaron radical y rápidamente los abordajes de salud pública. El objetivo de este trabajo consiste en delimitar preliminarmente el problema de las acusaciones y denuncias de persona a persona en contextos de proximidad bajo el régimen del confinamiento en el área metropolitana de Buenos Aires (AMBA). En este trabajo se examinan tres cuestiones: 1) las perspectivas antropológicas sobre la judicialización; 2) las amplias consecuencias y efectos paradojales que las acusaciones, denuncias y judicializaciones han demostrado tener para el bienestar, la salud y la supervivencia de las personas y conjuntos sociales afectados; y 3) las particularidades que asume el trabajo antropológico en contextos altamente normatizados, sancionados y criminalizados. Este artículo se estructura respecto del caso del AMBA, para

* El artículo es parte de las investigaciones que he realizado durante las últimas décadas sobre antropología de la salud en zonas de marginación social. Algunos de los temas que anteriormente he abordado han sido: epidemia por el VIH, consumos problemáticos de drogas, tratamientos psi, entre otros. Finalmente, el artículo forma parte del estudio “Tecnologías Terapéuticas 'Psi', Malestares y Vida Cotidiana en Poblaciones Marginalizadas del Área Metropolitana de Buenos Aires”, Proyecto Ubacyt, UBA (2018-2021) (20020170100239BA).

** Doctora en Ciencias Naturales de la Universidad Nacional de La Plata y Licenciada en Antropología de la Universidad Nacional de La Plata. Investigadora Principal del Consejo Nacional de Investigaciones Científicas y Técnicas, Conicet, Argentina. Profesora Asociada de la Universidad de Buenos Aires, Argentina. Entre sus últimas publicaciones están: "When Life Becomes a Burden at the Urban Margins of Buenos Aires”, Medical Anthropology 39, n. ${ }^{\circ} 2$ (2019): 153-166, https://doi.org/10.1080/01459740.2019.16 67993; "On Ethnographic Positions in the Anthropology of Health in South America”, Salud Colectiva 13, n. 3 (2017): 359-373, https://doi.org/10.18294/sc.2017.1104 $ه$ mariaepele33@gmail.com 
cuyo análisis se ensamblan materiales heterogéneos: antecedentes históricos, argumentos antropológicos, resultados de investigaciones, información de medios de comunicación, normativas, etc. Específicamente, el texto focaliza en aquellas prácticas desarrolladas durante los meses del período inicial de confinamiento en el AMBA, desde el 20 de marzo hasta el 9 de noviembre 2020. El análisis expuesto en este artículo arroja luz sobre los modos en que las acusaciones y denuncias en contextos de proximidad no solo fragilizan las tramas sociales, profundizan las desigualdades y acentúan la incertidumbre sobre las respuestas sociales frente a la posible infección y muerte, sino que también comprometen y distorsionan las estrategias epidemiológicas preventivas y de cuidado. Si bien acciones legales del mismo tipo han tenido lugar para otras dolencias y epidemias, las acusaciones y denuncias en contextos de proximidad, en este caso, son entendidas como estrategias habilitadas y legitimadas que expresan y traducen a la microescala del aislamiento profundas transformaciones de los vínculos entre salud pública, legislación y fuerzas de seguridad en los regímenes del confinamiento social.

Palabras clave: antropología de la salud, formaciones jurídico-sanitarias, judicialización, lógica de acusaciones, pandemia por el SARS-CoV-2.

\section{The Multiplication of Penalties: Preliminary Issues in Dealing with Proximity Accusations and Allegations in Times of Pandemic}

Abstract: With the outbreak and devastating spread of the SARS-CoV-2 pandemic, different forms of containment methods have been implemented across Latin America that have radically and rapidly transformed public health approaches. The purpose of this paper is to provide a preliminary delimitation of the problem of person-to-person accusations and complaints in proximity contexts under the confinement regime in the metropolitan area of Buenos Aires (AMBA). This paper examines three issues: 1) anthropological perspectives on judicialization; 2) the broad-ranging consequences and paradoxical effects that accusations, denunciations, and judicializations have been proven to cause for the well-being, health, and survival of affected individuals and social groups; and 3) the particularities that anthropological work assumes in highly normatized, sanctioned, and criminalized contexts. This article addresses heterogeneous materials: historical background, anthropological arguments, research results, media information, regulations, etc., to analyze the case of the AMBA. Specifically, the text focuses on the practices employed during the months of the initial period of confinement in AMBA, from March 20 to November 9, 2020. This analysis sheds light on the ways in which proximity accusations and allegations lead to the fragmentation of social fabrics, deepen inequalities, and accentuate uncertainty about social responses to possible infection and death. They also compromise and distort 
epidemiological, preventive, and care strategies. While legal actions of the same type have taken place for other ailments and epidemics, proximity accusations and allegations, in this case, are understood as enabled and legitimized strategies that express and translate deep transformations - at the micro-scale of isolation - of the links between public health, legislation and security forces in social confinement regimes.

Keywords: Anthropology of health, judicialization, legal and public health formations, logic of accusation, SARS-CoV-2 pandemic.

\section{A multiplicação das penas: questões preliminares para a abordagem de acusações e denúncias de proximidade em tempos de pandemia}

Resumo: com a irrupção e o devastador avanço da pandemia ocasionada pelo Sars-CoV-2, foram implementadas diferentes formas de confinamento que, na América Latina, transformaram radical e rapidamente as abordagens de saúde pública. O objetivo deste trabalho é delimitar preliminarmente o problema das acusações e das denúncias de pessoa a pessoa em contextos de aproximação sob o regime do confinamento na região metropolitana de Buenos Aires (RMBA). Neste trabalho, foram examinadas três questões: 1) as perspectivas antropológicas sobre a judicialização; 2) as amplas consequências e os efeitos paradoxos que as acusações, as denúncias e as judicializações vêm demonstrando ter para o bem-estar, a saúde e a sobrevivência das pessoas e dos conjuntos sociais afetados; 3) as particularidades que o trabalho antropológico assume em contextos altamente normatizados, sancionados e criminalizados. Este artigo trata do caso da RMBA, no qual a análise é estruturada a partir de materiais heterogêneos: antecedentes históricos, argumentos antropológicos, resultados de pesquisas, informação de meios de comunicação, leis etc. especificamente, no texto, foca-se naquelas práticas desenvolvidas durante os meses do período inicial de confinamento na RMBA, desde 20 de março até 9 de novembro de 2020. A análise exposta no artigo esclarece acerca dos modos pelos quais as acusações as denúncias em contextos de aproximação não somente fragilizam as relações sociais, aprofundam as desigualdades e acentuam as incertezas sobre as respostas sociais diante da possível infecção e morte, mas também comprometem e distorcem as estratégias epidemiológicas, preventivas e de cuidado. Embora as ações legais do mesmo tipo venham tendo lugar para outras doenças e epidemias, as acusações e as denúncias em contextos de aproximação, nesse caso, são entendidas como estratégias habilitadas e legitimadas que expressam e traduzem à microescala do isolamento profundas transformações dos vínculos entre saúde pública, legislação e forças de segurança nos regimes do confinamento social.

Palavras-chave: antropologia da saúde, formações jurídico-sanitárias, judicialização, lógica de acusações, pandemia pelo Sars-CoV-2. 
Las penas y las vaquitas, se van por la misma senda, las penas son de nosotros,

las vaquitas son ajenas. Atahualpa Yupanki (El arriero, 1944)

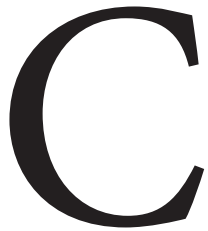
on la irrupción de la pandemia por el SARS-CoV-2 y frente al devastador avance que se venía generando en los países que no habían tomado medida alguna, o que no las habían tomado a tiempo en Latinoamérica, se desarrollaron políticas que, bajo las condiciones de emergencia, resonaban o replicaban parcial o totalmente algunas de las aplicadas en el norte global. En ciertos países de la región, entre ellos en Argentina, se implementaron diferentes disposiciones de confinamiento. Algunos de estos regímenes han estado regulados por una inédita amalgama de normativas sanitarias, políticas, legales, militares y de seguridad. Esta combinación discontinuaba, e incluso se oponía, a saberes establecidos respecto a la prevención y la salud pública latinoamericana como, por ejemplo, a los generados durante la pandemia por el VIH (Abadía 2004; Castro y Farmer 2003; Iriart et al. 2002). Las medidas y normativas de los confinamientos y otros abordajes en salud pública han mostrado y profundizado ciertas orientaciones y procesos generales. Por un lado, se han registrado procesos como: la rápida medicalización de todo aspecto de la vida social, la popularización forzada de rudimentos de epidemiología para vigilar y regular los contextos de proximidad y el desarrollo de algunas campañas basadas en el miedo y en discursos de estigmatización y culpabilización individual de la infección y muerte (Basile 2020). Por otro lado, se han profundizado las crisis económicas preexistentes, la desigualdad y la pobreza, específicamente en los pueblos originarios (Aljanati et al. 2020). Asimismo, la delegación del control y represión de cuestiones sanitarias a las fuerzas de seguridad ha acentuado la tradición de abuso, violencia y muerte que su ejercicio ha tenido por décadas (Meyer 2020; Tiscornia 2004). Además, la escalada de controversias y disputas respecto a las normativas reprodujeron y radicalizaron las oposiciones políticas y partidarias preexistentes. Por lo tanto, conjuntamente con la devastadora expansión de la pandemia, estos procesos y abordajes han modificado radical y aceleradamente tanto los contextos de vida y de cuidado cotidianos, como los procesos de salud-enfermedad-atención y de morir, lo que hace difícil subsumirlos a los argumentos académicos disponibles.

Dentro de este inabordable y abrumador escenario, este trabajo busca delimitar un problema en apariencia mínimo y marginal. Estoy haciendo referencia al rápido incremento de la vigilancia, acusación y denuncia de persona a persona relativas a infracciones de las disposiciones y/o sospechas de potenciales peligros de infección o contagio de otros, que fueron contaminando los vínculos de proximidad bajo el régimen del confinamiento en el área metropolitana de Buenos Aires (AMBA). Noticias de acusaciones y denuncias similares ocurridas en otros países y regiones habían 
llegado antes de que la pandemia afectara con virulencia el cono sur (González 2020; Gonzalo 2020). Asimismo, este tipo de acciones guardan semejanza con dinámicas registradas en otras dolencias y epidemias, como por el VIH-SIDA (Farmer 1999, 1992). Sin embargo, en esta ocasión se generó una explosión de dichas acciones, sobre todo durante los primeros meses del confinamiento. Este acelerado incremento de acusaciones ocurrió con respecto a una dolencia altamente contagiosa, significativamente mortal y cuando todavía se desconocía con precisión sus modos de transmisión, evolución, tratamiento y prevención. El aumento acelerado de estas acciones de acusación y denuncia sucedió en sincronía con el rápido incremento de infracciones y arrestos por parte de fuerzas de seguridad. Además de su escala de crecimiento, estas prácticas fueron inéditas en la legitimación, promoción oficial y lógicas jurídico-penales que fomentaron y regularon su ocurrencia. La importancia de delimitar y examinar preliminarmente este problema no reside únicamente en que dichas prácticas fragilizan las tramas sociales, profundizan las desigualdades y segregaciones preexistentes y acrecientan la distancia, desconfianza e incertidumbre en torno a las respuestas ante una eventual infección, enfermedad y/o muerte. La importancia de delimitar este problema consiste en que, al convertirse en anclajes de los modos de gobiernos de la emergencia, estas prácticas de acusación y denuncia de persona a persona comprometen el desarrollo de ciertas estrategias epidemiológicas preventivas y de cuidado (rastreos, contactos, etc.) debido a la lógica punitiva-sanitaria que las regulan.

El artículo examina tres cuestiones preliminares para el abordaje de la lógica acusatoria y de denuncia de proximidad en tiempos de pandemia. Partiendo del análisis de las perspectivas antropológicas sobre la judicialización (Comaroff y Comaroff 2006; Foucault 2006; Tiscornia, Kant de Lima y Eilbaum 2009), en primer lugar, se realiza un bosquejo preliminar de las especificidades de las lógicas acusatorias. Dicho bosquejo emerge al situarlas en el mapa de los modos de judicializar y penalizar en salud y de las formaciones jurídico-sanitarias que la antropología ha investigado (Biehl 2013; Epele 2007; Menéndez 2009; Molina 2018). En segundo lugar, se problematiza brevemente las amplias consecuencias y efectos paradojales que estos procesos han demostrado tener para el bienestar, la salud y la supervivencia de las personas y grupos humanos afectados (CELS 2016; Epele 2008; Grimberg 2005). Por último, se exploran las particularidades que asume el trabajo antropológico en contextos altamente normatizados, sancionados y criminalizados, no solo para el montaje de dispositivos preventivos y de cuidado, sino también para la adecuación de sus métodos y técnicas para llevarlo a cabo (Bourgois, Lettiere y Quesada 1997; Epele 2010).

Desde el análisis preliminar de estas cuestiones las acusaciones y denuncias en contextos de proximidad son entendidas como estrategias habilitadas y legitimadas oficial, pública y jurídicamente. Estas estrategias expresan y traducen a la microescala del aislamiento rápidas y profundas transformaciones de los vínculos entre salud pública, legislación penal y fuerzas de seguridad en los regímenes del 
confinamiento social. A diferencia de los abordajes de salud pública de tradición latinoamericana y de los modos de judicializar en salud dominantes en el sur global, esta formación sanitaria-jurídico-policial combina tres dimensiones: 1) universaliza normativas sanitarias a escala nacional relativas a la transmisión de la infección y las interpela desde figuras del código penal; 2) hace del sujeto individual el foco privilegiado de la "peligrosidad" y de los daños en la salud pública como un todo; y 3) delega a las fuerzas de seguridad el poder de controlar, reprimir y sancionar (Basile 2020). Con estas transformaciones se redistribuyen socialmente las penas y se hibridiza y subordina lo sanitario a la seguridad. Debido a lo anterior, se normalizan las sanciones, denuncias y judicializaciones, en lugar de situarlas como figuras extremas y aplicadas bajo condiciones excepcionales.

Ahora bien, es indiscutible la importancia de la implementación temprana de disposiciones de prevención (confinamientos, aislamientos, distancia social, rastreos y testeos de casos, etc.), campañas de información respecto del cuidado y provisión de asistencia en el sistema de salud, social y económica frente a la catástrofe sanitaria que la pandemia ha desencadenado. Sin embargo, esta importancia no llega a ser mayor que la relevancia que tienen las características, prioridades y consistencia de las estrategias preventivas, los tipos de sanciones y legislaciones que se incluyen, las instituciones a las que se delega el poder de su regulación y la sensibilidad de las disposiciones respecto a las condiciones económico-políticas de diferentes contextos sociales.

Hay un acervo social extenso y sofisticado al que se podría volver para anclar los abordajes, para revisar y avanzar, para reencontrar el camino que parece haberse extraviado. Este acervo está constituido por: 1) las perspectivas de salud pública de orientación latinoamericana, 2) la atención experta y dedicada de los profesionales de salud frente a la infección, enfermedad y muerte, 3) las acciones en organizaciones sociales locales orientadas a la subsistencia, al cuidado y la supervivencia y 4) la amplia experiencia latinoamericana en atención a catástrofes y emergencias (económicas, políticas, ambientales, humanitarias, entre otras). Por último, en lugar de escribir desde una posición de exterioridad que reproduce lo que se cuestiona, con este trabajo se revisa en tiempo real al desarrollo de la pandemia, la lógica acusatoria que nos revuelve y convoca desde diversas voces, rostros y enunciaciones. Este texto es una invitación a explorar territorios que eviten desencadenar otras catástrofes de devenir incierto y contribuyan a despejar horizontes compartidos de bienestar y salud.

\section{El abordaje}

El surgimiento y expansión de la pandemia por el SARS-CoV-2 estuvieron acompañados desde un principio por estudios e investigaciones en ciencias sociales que indagaban por sus aspectos más relevantes. Inicialmente, nociones como la sindemia y el hecho social total se convirtieron en referencias ineludibles para dar 
cuenta de las características inéditas de esta pandemia (Horton 2020; Singer 2008; Ventura, Pontes y Coimbra 2020). Uno de los aspectos dominantes en los primeros estudios es la relevancia de las desigualdades —económicas, laborales, de vivienda, de género, de acceso al sistema de salud - en la gravedad y muerte de los afectados. Al cambiante y continuo desarrollo de los saberes biomédicos y epidemiológicos, se les agregó la incertidumbre sobre los efectos de las diferentes políticas públicas y sanitarias implementadas a nivel local y global. Debido a las características de la pandemia por el SARS-CoV-2 y de las diferentes normativas preventivas que se aplicaron para regular y controlar la vida de las poblaciones, las metodologías y técnicas de investigación tuvieron que adaptarse creativamente tanto en la etapa de registro como en los análisis de los materiales disponibles y de los recursos habilitados para llevarlas a cabo (encuestas, entrevistas virtuales, sitios web, redes sociales, entre otros).

Con el fin de delimitar el problema de las acusaciones y denuncias en contextos de proximidad, este artículo se estructura en relación al caso del área metropolitana de Buenos Aires (AMBA), a través del análisis de las cuestiones preliminares. En la delimitación y análisis del problema se integran y ensamblan materiales heterogéneos: antecedentes históricos, argumentos antropológicos, resultados de investigaciones, información de medios de comunicación, normativas, etc. Esta alternativa metodológica se impone para respetar las condiciones excepcionales de producción de este texto. Vale la pena aclarar que la temática bajo examen es difícil investigarla con las tecnologías disponibles durante el aislamiento. En este caso en particular, los abordajes y técnicas de investigación formarían parte del problema mismo a examinar, pues el uso de ciertas tecnologías para su indagación puede exponer a los sujetos a las mismas lógicas punitivas que se analizan.

Las fuentes de la información y estadísticas relativas a la salud han sido elaboradas y publicadas por sitios oficiales de gobiernos (nacionales, provinciales, municipales). Las informaciones relativas a las denuncias de proximidad fueron publicadas y difundidas a través de los medios de comunicación, específicamente a través de los periódicos y la televisión. En los medios se informó que sus fuentes estaban basadas en organismos oficiales (ministerios y secretarías de seguridad, de justicia, nacionales, provinciales y de la ciudad de Buenos Aires).

Los materiales e informaciones que conforman el corpus de este trabajo, refieren principalmente al área metropolitana de Buenos Aires, que articula la ciudad de Buenos Aires y el Gran Buenos Aires (provincia de Buenos Aires). Temporalmente el artículo se basa en la primera etapa del aislamiento preventivo y obligatorio (ASPO), que tuvo lugar desde el 20 de marzo al 9 de noviembre de 2020 en esta región, y hasta el 30 de noviembre 2020 en otras regiones del país. Para el comienzo del ASPO, el total de casos era 225 y hubo cuatro personas fallecidas. Para el fin de esta etapa del ASPO el total de casos sumaban 1424533 y 38730 personas fallecidas (Ministerio de Salud de Argentina 2020). 


\title{
Sobre acusaciones, sanciones y denuncias
}

\author{
Lo que nos hizo dichoso \\ tal vez se pueda olvidar. \\ Los años en su pasar \\ mudarán los pensamientos, \\ pero angustias y tormentos \\ son marcas que han de durar. \\ Atahualpa Yupanqui (El payador perseguido, 1964)
}

La rápida expansión de las diversas disposiciones para detener, menguar o ralentizar la pandemia por el SARS-CoV-2 en países de Latinoamérica ha incluido aparatos pedagógicos, de vigilancia, de rastreo y testeo de casos y de intervención sobre los afectados. Estos aparatos discursivos y de intervención, con diferentes gramáticas, oficializaban las normativas de prevención de modo universal y bajo la fórmula del imperativo. Su objetivo era garantizar la provisión de la atención médica, la subsistencia alimentaria y la supervivencia de aquellos sectores más expuestos a la infección y muerte. Las normativas incluyeron como tecnologías inherentes y estratégicas a su despliegue la vigilancia, denuncia, sanción moral y judicialización de las infracciones de las disposiciones y la delegación del poder de control a las fuerzas de seguridad y a otros representantes de instituciones del Estado.

La proliferación de denuncias públicas contra diferentes organismos del Estado por parte de personas y colectivos en zonas de marginación del área metropolitana de Buenos Aires tuvo lugar desde los inicios mismos de la pandemia. Estas se explican debido a la compleja combinación entre la profundización de la crisis económica preexistente y el confinamiento social. La mayor parte de las denuncias se focalizaron en las condiciones y disposiciones sanitarias y en la actuación de las fuerzas de seguridad en el ámbito de salud. A su vez, las denuncias tenían una correspondencia con fórmulas previas y locales de reclamo. Tanto las unas como las otras se refieren a la desigualdad en el acceso al sistema de salud, la falta de atención y/o de insumos médicos, las precarias condiciones materiales de vida (falta de agua, alimentos, recursos para la higiene, etc.), los abusos de poder y la violencia institucional por parte de fuerzas de seguridad ${ }^{1}$.

Sin embargo, estas acusaciones y denuncias a los gobiernos quedaron relegadas y sepultadas por la exposición pública en medios de comunicación de miles de infracciones diarias de las normativas, en las que las fuerzas de seguridad cobraron

1 Se puede ver algunos ejemplos sobre denuncias públicas por falta de insumos médicos, falta de agua y recursos y abusos y muertes por fuerzas de seguridad en las páginas web de los siguientes medios: La Nación, Perfil, Página 12, Infobae y Clarín. 
un protagonismo central ${ }^{2}$. La inundación de los espacios y medios públicos con estas infracciones redujeron la visibilidad de aquellas otras dinámicas más relevantes y urgentes (desigualdad en el acceso al sistema de salud, falta de cuidado y de atención respecto a otras enfermedades, desmantelamiento de economías y estrategias informales de obtención de recursos, desempleo, etc.) que se iban generando en los sótanos de la vida social bajo el régimen de confinamiento. En este complejo escenario fueron expuestos y diseccionados públicamente ciertos casos como ejemplos de peligrosidad, diseminación de la infección y amenaza a la salud pública; la mayoría se referían a infracciones de movilidad y reunión social. La exposición de los casos se convirtió en una suerte de modelo para armar en el que se aplicaban: 1) los saberes fragmentarios y cambiantes sobre el SARS-CoV-2, 2) las normativas, sanciones jurídicas y formas de penalización respecto a la infección real o posible, 3) la actuación de las fuerzas de seguridad y 4) los modos de responsabilizar, calificar y actuar sobre aquellas personas que eran susceptibles de padecer la infección y/o de transmisión del virus (Angulo 2020). La repetición pedagógica de estos casos fue extrañamente cubierta con formatos y contenidos semejantes por autoridades y agencias comunicativas con diferentes intereses político-sociales. Desde diferentes sectores e instituciones, se invitaba a su reproducción formal e informal por diversos sectores sociales.

Una ola de prácticas y discursos de acusación y denuncia de persona a persona fue creciendo en los contextos de proximidad debido al potencial peligro de contagio a otros, sospecha de infección o infección en curso. En su difusión en los medios de comunicación, estas prácticas decían ser realizadas por sujetos individuales y colectivos: vecinos, personas cercanas y (des)conocidas en medio de los particulares contextos del confinamiento social. Entre las acciones que empezaron a hacer los grupos y las personas encontramos: la observación y vigilancia de otros, el señalamiento y acusación interpersonal de casos sospechosos, el escrache y exposición pública sin preservación de la identidad de afectados (en redes sociales, medios de comunicación, etc.), la denuncia de otros por los diferentes canales habilitados por

2 Las cifras de las infracciones diarias de normativas, acusaciones y denuncias fueron omnipresentes en los medios de comunicación al inicio del confinamiento (Caram 2020). Por ejemplo, cuando para el 1 de abril del 2020, a diez días del ASPO, el total de casos acumulados por Covid-19 era de 1133 y las personas fallecidas sumaban 32 (Ministerio de Salud de Argentina 2020), ya se registraban 728862 infracciones y 21642 detenciones por parte de las fuerzas federales; 16219 intervenciones por la policía de la Provincia de Buenos Aires; 3031 infracciones y 380 detenciones por las fuerzas de la Ciudad de Buenos Aires. Además, para esa fecha se registraron 38313 denuncias a la línea habilitada para la ciudadanía por violación de la cuarentena nacional, 11293 en la ciudad de Buenos Aires (“Cuarentena en cifras" 2020). Para esa fecha, los medios informaron que se registraron algo más de 8500 imputaciones de los artículos 205 y 209 del Código penal (Lavieri 2020).

A casi dos meses de la implementación, el 14 de mayo, había 7134 casos acumulados y 353 personas fallecidas en el país (Ministerio de Salud de Argentina 2020). Al mismo tiempo las fuerzas federales notificaron a 8521931 personas por infracción de las disposiciones y detuvieron a 84565 . En la ciudad de Buenos Aires, fueron demoradas, notificadas o trasladadas a su domicilio 22916 y 1463 detenidas; solo en la ciudad, ya se había acumulado 30695 denuncias en la línea habilitada por incumplimiento de la cuarentena ("Más de ocho millones" 2021). 
las autoridades en organismos de gobierno a tal fin, las amenazas de realizar denuncias, las denuncias judiciales efectivas e incluso las contradenuncias de los afectados. Solo las denuncias y amenazas contra los profesionales de la salud fueron objeto de intervenciones legales orientadas a su protección frente a la propagación de estas prácticas (Zunino 2020). El lenguaje y las fórmulas de estas acusaciones, son versiones modificadas de los discursos oficiales y las disposiciones normativas. La reproducción, sofisticación y profundización de las acusaciones y denuncias, incluyeron desde ataques y daños materiales a los afectados, la expulsión forzada de personas y familias afectadas de viviendas y/o barrios, hasta las consideradas bromas por falsas denuncias a personas no afectadas o personas no existentes ${ }^{3}$. En un contexto de más de $40 \%$ de pobreza, $10 \%$ de indigencia y desigualdad social y económica extrema, las presentaciones públicas de estos modelos estaban orientadas a una particular lógica de cuidado atravesada por lo punitivo. La lógica imperante era "servir a los gobiernos a cuidarnos" a través de la denuncia de otros, complementada con el cuidado de sí, como forma privilegiada de cuidar a otros. Considerando los cambios de características y desafíos en la dinámica de la pandemia, estas prácticas se fueron desdibujando en los medios a mediados del 2020 cuando las cifras de infección, muertes diarias y acumuladas cobraron centralidad, aunque persistieron bajo otras formas e intensidad hasta el presente.

Aunque inéditos en sus características, escalas y formas de articularse entre sí, algunos de estos procesos han operado en diferentes problemas de salud y epidemias (Crawford 1994; Farmer 1992; Grimberg 1995; Pecheny 2001). Sin embargo, a diferencia de la pandemia por el SARS-CoV-2, en otras epidemias y dolencias tanto las acusaciones, denuncia y judicialización, como la estigmatización y discriminación que expresan, han sido objeto de claras y tajantes intervenciones por parte del Estado, del activismo en salud y de organismos no gubernamentales para proteger a los sujetos y conjuntos de personas afectados por estas prácticas.

La problematización antropológica del creciente protagonismo de la judicialización y de la cultura de la legalidad en diferentes órdenes de la vida social ha incluido inevitablemente el campo de la salud (Comaroff y Comaroff 2006). Una amplia literatura antropológica se ha producido acerca de los modos de judicializar problemas específicos de salud y de las formaciones jurídico-sanitarias creadas en Latinoamérica durante las últimas décadas (Biehl 2013; Parker 2009). Cuando las denuncias y judicialización han entrado en escena en este campo la orientación general ha sido las demandas contra Estados, instituciones, empresas e instituciones de salud. Además, aquellas denuncias y judicializaciones que se focalizaron en sujetos individuales mayormente han tenido lugar en la penalización de problemas de salud (consumo problemático de drogas, aborto, etc.) o, más recientemente, en litigios entre personas por transmisión de enfermedades infecciosas. Tanto en un caso como en el otro estas acusaciones hacia sujetos han sido ampliamente cuestionadas

3 Periódicos consultados: La Nación (https://www.lanacion.com.ar/), Perfil (https://www.perfil.com/), Página 12 (https://www.pagina12.com.ar), Infobae (https://www.infobae.com) y Clarín (https://www.clarin.com/). 
en su eficacia, objetivos y consecuencias para los derechos humanos, la salud y la supervivencia (Inchaurraga 2003; Tarducci y Daich 2018; Unaids 2013).

Situar dentro de esta breve cartografía el problema de las acusaciones, sanciones morales y denuncias de persona a persona en el contexto del confinamiento social hace posible delinear de forma preliminar algunas de las cuestiones sobre sus características inéditas. En primer lugar, las técnicas de acusación y denuncia en los contextos de proximidad se convirtieron en estrategias habilitadas y legitimadas oficial, jurídica y públicamente. Estas técnicas, por demás, se corresponden con las tecnologías de vigilancia, sanción y judicialización inherentes a la formación sanitaria-jurídica-policial que fundó los confinamientos. En segundo lugar, en estas técnicas de acusación y denuncia confluyeron la popularización y traducción simplificada de saberes biomédicos y epidemiológicos incompletos, parciales, contradictorios entre sí y en desarrollo incierto. En los casos de las denuncias, estos saberes fueron filtrados por la codificación de la legislación penal. La apropiación colectiva y subjetiva del idioma epidemiológico y jurídico y de las prácticas de vigilancia serían una suerte de privatización de los idiomas y prácticas usadas por el Estado para justificar, controlar, denunciar y sancionar cuestiones relativas a la infección, enfermedad, vida y muerte. Además, estas prácticas inevitablemente comprometieron -o fueron contraproducentes - a las acciones preventivas y de cuidado.

Resumiendo, el desarrollo de estas prácticas expresa y traduce en la microescala del aislamiento una rápida y profunda reorientación de los vínculos entre salud pública, sanciones morales, legislación penal y fuerzas de seguridad, con una incierta y progresiva evolución respecto de la salud y la sociabilidad en general. En lugar de continuar las políticas y tradiciones de salud pública usadas anteriormente en Latinoamérica, estos abordajes mezclan e incluso subordinan lo sanitario a las cuestionadas fuerzas de seguridad locales. A través de esta subordinación se universaliza a escala nacional las tradiciones que durante décadas se han malformado en la gestión de las poblaciones en zonas de marginación social.

\section{Sobre la ley y la judicialización}

Desde décadas atrás, la ley, la ilegalidad y la transgresión, han sido problemas recurrentes en los argumentos que buscan explicar las complejas realidades en Latinoamérica y Argentina (Foucault 2006; Nino 2005; O’Donnell 2002). Sin embargo, la creciente relevancia de la ley, de los aparatos jurídico-legales y de la judicialización respecto a diferentes órdenes de la vida social han adquirido mayor visibilidad en los abordajes antropológicos en el sur global (Epele 2008a; Tiscornia, Kant de Lima y Eilbaum 2009). La judicialización, es decir, el proceso de subsunción bajo el aparato jurídico de diferentes áreas de experiencia social (saberes, idiomas, prácticas, lógicas, sujetos, etc.) que antes eran reguladas por otras prácticas e instituciones, ha cobrado central relevancia en los argumentos antropológicos contemporáneos. De acuerdo con Jean y John Comaroff (2006), la creciente importancia de la ley y la 
judicialización en el sur global, paradójicamente acompañada de un desorden generalizado, se manifiesta en la constante referencia a las leyes y a tecnologías jurídicas en diversos procesos y experiencias, desde los discursos públicos hasta la lucha de minorías sociales. Esta reorientación la denominan cultura de la legalidad, noción que refiere a la creciente apelación a la ley, lo judicial y sus ficciones de poder como una instancia para dirimir conflictos no solo entre personas, sino en los vínculos con los estados, el mercado, los gobiernos y diversas instituciones (escolares, de salud, etc.). Además, sostienen que la cultura de la legalidad y la judicialización en clara expansión pareciera ofrecer un patrón simple de conmensurabilidad de experiencias, dolencias y daños heteróclitos. Dicha cultura individualiza y desagrega las tramas sociales y transforma a las personas y colectivos en sujetos legales, entendidos en términos de lenguajes y categorías jurídicas, jurisprudencias y jurisdicciones nacionales y/o globales. Los vínculos sociales, próximos, íntimos y hasta de los sujetos consigo mismos quedan impregnados tanto con estos lenguajes y técnicas jurídicas, como con las moralidades, penas y culpas que multiplican (Comaroff y Comaroff 2006).

El campo de la salud no ha sido una excepción en la avanzada de la legalidad y la judicialización. Desde hace años se vienen forjando nuevos vínculos entre problemas de salud, la biomedicina, los mercados de tecnologías en salud, las legislaciones, el aparato de justicia y las fuerzas de seguridad. Sin embargo, estas articulaciones no son algo nuevo. Mientras que algunos dominios de salud/enfermedad han registrado una intensiva articulación - si no superposición - de lo jurídico y lo sanitario, otros continuaron subsumidos solo a la biomedicina y/o prácticas legas tradicionales. En esta genealogía, a su vez, se dibujan períodos históricos de mayor asociación y naturalización de los vínculos entre los modos de abordaje jurídico-legales y de salud, frente a otros de máxima crítica y diferenciación entre los campos.

Diferentes estudios han señalado que cuando ciertas dolencias se subsumen al campo jurídico-legal surgen dificultades inherentes a este proceso. Por un lado, encontramos el proceso de traducción y codificación de los saberes expertos (biomédicos, psiquiátricos y/o psicológicos) en términos de facticidad jurídica y en un régimen de verdad legal (Foucault 2005). Dicha traducción modifica las condiciones epistémicas, ontológicas y de género que los había modelado previamente (Petryna 2002). Por otro lado, incluye la transformación de, primero, los sujetos reales en sujetos codificados por la ley y, segundo, la determinación del grado de conocimiento que las personas tienen sobre las dolencias en cuestión y las leyes y las normativas que las regulan. Los ocasionales obstáculos en esta traducción y codificación surgen del carácter fragmentario, e incluso contradictorio, de ciertos saberes biomédicos y de las dificultades de probar en términos jurídicos que el sujeto en cuestión no solo dispone de la información, sino que ha tenido un comportamiento identificable y categórico en relación a la dolencia (Biernat 2018).

Desde los estudios sociales de la ciencia, estas dificultades en la traducción de evidencia empírica en prueba jurídica son consideradas como algo inherente a la 
producción del conocimiento científico. Ahora bien, dicha dificultad resulta más evidente cuando emerge una nueva dolencia o enfermedad. Como se ha señalado, el conocimiento científico es indisociable de: 1) la tecnología que interviene en su producción, 2) las políticas que lo legitiman e instituciones que lo validan, 3) las lógicas de poder que lo subsumen, 4) las respuestas sociales que genera, 5) las economías y los mercados que lo regulan y 6) los modos en que las poblaciones demandan tanto por daños en la salud, como por las eventuales muertes en contextos específicos (Petryna 2002). Así, los modos de judicializar comportamientos vinculados a problemas de salud, no solo son complejos y de incierta evolución, sino que desde hace décadas se han mostrado mayormente indecidibles. Por ejemplo, los análisis históricos hechos frente a la infección de sífilis bajo el higienismo de la década de 1930 mostraron que las judicializaciones hechas por varones a trabajadoras sexuales generalmente no llegaron a ningún destino, entre otras cosas porque no se podía probar que las mujeres conocían el mecanismo de transmisión (Biernat 2018). Las perspectivas acerca de las dolencias que elaboran los sujetos no se corresponden linealmente con los saberes biomédicos, sino que estos son traducidos y reapropiados bajo las coordenadas de los modos de vivir, entender y sufrir bajo tradiciones locales y contextos histórico-económico-políticos particulares (Margulies 2014).

Cuando el aparato jurídico-penal entra en juego en el campo de la salud, la complejidad de los procesos y de sus consecuencias se multiplican. En aquellos casos en que las disposiciones de confinamientos para regular la pandemia por el SARS-CoV-2 contemplan normativas que refieren a figuras del código penal, la situación se complejiza a niveles desconocidos y con un devenir por completo incierto. Por un lado, la delegación a las policías y fuerzas de seguridad de la gestión de los confinamientos han mostrado todo tipo de los abusos y violencias ya conocidos en esta región (abusos de autoridad, gatillo fácil, etc.) (Tiscornia 2004). Por otro lado, el conocimiento biomédico sobre el SARS-CoV-2 era - y sigue siendo- incompleto, cambiante y en permanente evolución. Aunque la judicialización se realice o sea potencial la referencia a la penalidad y a la criminalización ha inaugurado nuevos modos de anudar lo jurídico-sanitario-policial, que trastocan y exceden los modos habituales de judicializar en salud. Particularmente, estas formaciones han contaminado las relaciones de proximidad en vez de nutrirlas y multiplicarlas con otros lazos, idiomas y prácticas que, de hecho, han sido los reales protagonistas de la asistencia y supervivencia bajo el régimen de confinamiento.

\section{Modos de judicializar en salud}

Como se ha mencionado anteriormente, el sostenido y creciente estrechamiento y diversificación de los vínculos entre los aparatos legales-jurídicos y problemas de salud ha sido investigado por la antropología. En ciertos estudios sobre problemas de salud se han propuesto algunas formaciones jurídico-sanitarias que impregnan y modelan los modos de, primero, entender, prevenir, intervenir y tratar ciertas afecciones, enfermedades y formas de morir y, segundo, de reprimir las prácticas sociales 
que las generan. En estas formaciones participan de modo diverso y jerárquico. Por un lado, están los Estados-nacionales (instituciones de salud pública y políticas sanitarias, aparatos jurídico-legales, fuerzas de seguridad, etc.), organismos internacionales y conglomerados y empresas nacionales e internacionales de extracción, tecnologías biomédicas y productos farmacéuticos. Por otro lado, se encuentran las personas individuales, colectivos, organizaciones de la sociedad civil y minorías y movilizaciones sociales. En el campo de la antropología de la salud, una de las clásicas y más generalizadas formaciones jurídico-sanitarias, específicamente de las orientaciones críticas, consiste en integrar el problema de los derechos humanos en general y de la salud en particular en los argumentos que dan cuenta de los modos de vivir y padecer diferentes dolencias en condiciones de pobreza y desigualdad (Briggs y Mantini-Briggs 2003; Cueto 2009; Farmer 2003).

Por un lado, hay un conjunto de formaciones jurídico-sanitarias formuladas por investigaciones antropológicas en Latinoamérica que se caracterizan por demandar desde abajo, es decir, que son impulsadas por sujetos o colectivos, activistas, asociaciones y organizaciones de la sociedad civil hacia diversos organismos de poder (Estado, autoridades, instituciones de salud, empresas, etc.). Estas demandas desde abajo generalmente se focalizan en problemas relacionados con: 1) el acceso a recursos biomédicos y al sistema de salud, 2) derechos vulnerados, 3) cuestionamientos a la desigualdad, racialización y discriminación de ciertas políticas y prácticas y 4) la búsqueda de reparación y compensación de daños y muertes infligidos a sujetos, poblaciones indígenas y minorías sociales y políticas.

La epidemia por el VIH ha sido una prolífera cantera de formaciones jurídico-sanitarias problematizadas por la antropología en países de la región. Diversas formas de activismo y asociaciones participaron, por ejemplo, en la producción de legislaciones específicas que tuvieron como objetivos la protección de los derechos y la asistencia de los colectivos y personas afectadas, el acceso universal a la medicación, y la sanción por parte de las autoridades sanitarias a las instituciones que no cumplieran las normativas (Biehl 2007; Farmer 2003; Parker, Easton y Klein 2000) . $^{4}$ Estos reclamos y logros llegaron a ser paradigmáticos y se extendieron a otras dolencias y enfermedades.

Ahora bien, otro antecedente relevante han sido las denuncias y demandas por reparación de daños y muertes respecto de políticas y prácticas racializadas y eugenésicas de esterilizaciones masivas en poblaciones indígenas segregadas tanto en México como en Perú (Menéndez 2009; Molina 2018). Además, están aquellas formaciones jurídico-sanitarias relativas a problemas de salud como la tuberculosis en migrantes ilegalizados, tráfico de personas y trabajo esclavo en Argentina y Brasil (Goldberg 2013). Sumado a lo anterior, en las últimas décadas, la cuestión ambiental se ha convertido en un campo atravesado por variados modos de demandar, denunciar y judicializar las consecuencias para la salud de la megaminería, agronegocios,

4 Ver Ley Nacional de SIDA en: http://test.e-legis-ar.msal.gov.ar/leisref/public/showAct.php?id=6159 
petróleo, contaminación industrial y de residuos y falta de servicios básicos en zonas de marginación (Auyero y Swistum 2008; Svampa y Viale 2014).

Recientemente, se ha registrado un incremento de denuncias y modos de judicializar demandas realizadas por sujetos individuales o agregados contingentes de personas orientadas al acceso a servicios de salud, tratamientos (intervención, trasplantes, etc.) y principalmente productos farmacéuticos y nuevas tecnologías médicas (Biehl 2013). Debido a estos procesos, nuevas formas de medicalización y rejudicialización de la sexualidad han conllevado, por ejemplo, al acceso a recursos de salud relacionados con la epidemia por el VIH (Parker 2009). Estas transformaciones se relacionan con el avance de la desregulación neoliberal de las economías y políticas de salud, aunque, paradojalmente, bajo discursos progresivos y orientados al aumento de derechos, de ciudadanía.

Por otro lado, hay otro conjunto de formaciones jurídico-sanitarias en Latinoamérica problematizadas por la antropología que incluye aquellas en las que se demanda desde arriba. Estas se refieren a los casos en que se aplica la legislación vigente y los aparatos jurídicos-penales por parte de los Estados-nacionales en contra de las personas y poblaciones afectadas de ciertos problemas de salud, que los convierte en blanco judicial y de las fuerzas de seguridad. A nivel internacional, esta judicialización desde arriba también se ha registrado entre Estados más poderosos y/u organismos internacionales contra aparatos estatales más débiles sobre las patentes de la industria farmacéutica, por ejemplo (Fassin 2007).

Sin embargo, entre los modos de denunciar y judicializar desde arriba más relevantes en los análisis antropológicos encontramos que en varias ocasiones las industrias, organismos del Estado o clínicas que despliegan los aparatos jurídico-penales respecto al daño de la salud pública están respondiendo a demandas y denuncias por problemas de salud interpuestas por individuos o colectivos. Además, en ocasiones la legislación penal frente a ciertos problemas de salud incluye la intervención de las fuerzas de seguridad a cargo de la prevención-punitiva, es decir, tanto de la vigilancia, persecución y denuncia de las conductas sancionadas, como de la represión, judicialización y encierro. Entre las formaciones jurídico-penales desde arriba más trabajadas se encuentra el consumo problemático de drogas y el aborto (Epele 2010; Tarducci y Daich 2018)

Dentro de este conjunto de formaciones jurídico-sanitarias-penales se ubican las disposiciones para responder a la pandemia por el SARS-CoV-2 en numerosos países, algunos de ellos en Latinoamérica. En Argentina, los diferentes tipos de sanción por infringir las regulaciones del confinamiento varían desde multas hasta quince años de prisión de acuerdo con las figuras tipificadas del código penal. Además, el hecho de respaldarse en la ley penal hace que los problemas de salud y las personas que los padecen queden embebidos en las lógicas control y represión

5 Se hace necesario destacar la importancia para la salud pública, después de décadas de movimientos y luchas, de la sanción por parte del Congreso Nacional de la República Argentina de la Ley 27610 de interrupción voluntaria del embarazo (IVE), el 30 de diciembre del 2020. 
por las fuerzas de seguridad y por diferentes instituciones del Estado (salud, justicia, etc.). Las disposiciones y las sanciones de las infracciones se aplicaron a toda la población, sobre todo por faltas a las medidas de restricción de movilidad y reunión. Lo anterior atestigua una inversión radical de los usos habituales de la noción de “potencial peligro” y daño a la salud pública en las tradiciones locales ${ }^{6}$.

La rápida escalada de acusaciones, denuncias públicas y judiciales entre personas en contextos de proximidad durante la pandemia por el SARS-Cov-2 refiere a una reorientación radical de las políticas de salud pública. Dicha reorientación ha implicado una mimetización de las acciones por parte del Estado sobre los individuos en los contextos locales, con los aspectos legales y judiciales que edificaron los confinamientos y con la generalización a escala nacional de modos de gestión y gobierno de los cuerpos, de la vida y la muerte que por décadas habían dominado las zonas marginales.

\section{La multiplicación de las penas}

Cuando la legislación penal entra en escena con respecto a ciertos problemas de salud, los modos de vida de los sujetos afectados se ven contaminados por el aparato jurídico-penal-policial. La definición de los conceptos de penalización y criminalización elaboradas en investigaciones previas adquieren en este contexto una relevancia central. Por un lado, la penalización refiere a la denuncia y judicialización de prácticas y acciones relativas a ciertos problemas de salud cuando son subsumidas bajo la esfera de la legislación penal. Por lo tanto, la penalización implica una traducción e inclusión de lo sanitario a la lógica jurídica y de las fuerzas de seguridad. Por otro lado, la criminalización refiere a procesos sociales más complejos, amplios y de incierto devenir que transforman los modos de vivir, entender, expresar, vincularse, reconocerse a otros y a uno mismo, cuando ciertos dominios de vida y/o afecciones son subsumidas a la ley penal. La criminalización enfatiza las diferentes consecuencias que tiene la transformación de las coordenadas de referencia de ciertos padecimientos y de los conjuntos sociales afectados por los mismos, cuando quedan contaminadas por denuncias, sanciones morales, culpabilización, sospechas, denuncias y/o eventuales judicializaciones (Epele 2010). Al poner el foco en la criminalización, se exponen las maneras en que las lógicas de desigualdad política, económica, de género y de acceso a los sistemas de salud, modelan y orientan las direcciones de la sospecha, de la acusación, la denuncia, la sanción social y la impunidad en diversos contextos sociales y sanitarios (Epele 2007; Inchaurraga 2003).

Cuando se despliega la criminalización respecto a un problema de salud, este proceso tiene múltiples y amplias consecuencias para las personas afectadas y para los modos de entenderlo y abordarlo tanto por las instituciones como por la sociedad en su conjunto. Además, lejos de ser sujetos pasivos, las personas y los

6 Acerca de las controversias en Argentina en torno al criterio de legalidad de las disposiciones y las figuras del código penal ver: Gargarella (2020) y Pascual y Gasparini (2020). 
colectivos afectados, responden de diferentes modos frente a la acusación social, moral y legal. Estas respuestas de los sujetos suceden, aunque una judicialización efectiva no se dé en todos los casos, ni siquiera en la mayoría. Frente a la vigilancia y la posibilidad de sanción moral y penal, las personas afectadas desarrollan progresivamente modos alternativos a los legitimados por la ley. Estos se caracterizan por 1) ocultarse a las miradas de los otros, 2) transgredir las disposiciones, 3) refugiarse en zonas de marginación, 4) distanciarse socialmente de cualquier representante del Estado y personas que podrían acusarlos, 5) desplegar estrategias discursivas para comunicarse sin revelar cualquier información que desencadene en discriminación, estigmatización o sanciones morales y judiciales y 6) desarrollar prácticas y saberes informales de cuidado con diferentes niveles de (in)eficacia. Dichos saberes de cuidado en ocasiones integran algunos aspectos de las normativas con otros legos y de tradiciones populares. Es decir, a través de los saberes se contextualiza la normativa para hacerla sensible a las condiciones de vida (Epele 2008).

Las personas y conjuntos expuestos a la criminalización pueden ver comprometida su salud y supervivencia. Por lo tanto, estos sujetos individuales y colectivos desarrollan respuestas sofisticadas al sistema de salud y a las normativas sanitarias. Entre ellas encontramos: la desconfianza frente a los profesionales y el sistema de salud, las dudas acerca de los objetivos político-sociales de las normativas de cuidado, el deslizamiento e inversión del significado de los mensajes preventivos, la postergación de la consulta y la búsqueda de atención solo cuando las condiciones de la enfermedad se extreman. La relevancia variable de algunas de estas prácticas en determinados sectores sociales se corresponde con los modos históricos en que las lógicas de poder, los sistemas de salud y las fuerzas de seguridad han accionados sobre dichos sectores. El revuelto complejo entre prevención sanitaria-punitiva, rehabilitación represiva, encierros sufridos y dolencias criminalizadas facilita y favorece el gobierno de poblaciones en zonas de marginación. Al mismo tiempo, este complejo fragiliza la salud, vulnera los derechos y promueve la muerte-joven y evitable (Epele 2007).

Según la experiencia de décadas de la epidemia por el VIH, la apelación a la judicialización penal de la infección de persona a persona es considerada no solo como ineficaz sino también como contraproducente en materia de prevención y de cuidado de la salud. Dicha consideración es resultado de los análisis hechos por organismos internacionales como Unaids y la OMS en países tanto del norte global, como, aunque con solo pocos casos, en países el cono sur (Mannino 2015). De acuerdo con estos organismos, la criminalización tiene las siguientes consecuencias: 1) contradice los mensajes de salud pública respecto a la prevención y el cuidado, 2) establece una ruptura con el criterio de prevención basado en el supuesto de universalidad del posible contagio y por lo tanto del cuidado, 3) transforma la prevención en el control y vigilancia de grupos sociales específicos, lo que promueve la discriminación y estigmatización, 4) genera un sentido de seguridad ficticio y falso que está vinculado al desplazamiento de la responsabilidad del cuidado desde los vínculos sociales y de sí 
hacia la ley, la autoridad pública y las fuerzas de seguridad, 5) quiebra la confianza entre profesionales de la salud y pacientes, 6) promueve la evitación y/o rechazo de la realización de pruebas, diagnósticos y tratamientos respecto a la afección, 7) refuerza el estigma, la discriminación y la marginación laboral y social y 8) racializa las estrategias criminalizadoras y afecta con mayor extensión a las poblaciones marginadas y minorías (Onusida 2008; Unaids 2013).

En esta breve revisión de las consecuencias de la criminalización de problemas de salud, se puede destacar su carácter ineficaz y contraproducente respecto a los objetivos relativos a la salud pública. Esta criminalización en todos los casos favorece el sometimiento forzado a arbitrariedades de gobiernos y fuerzas de seguridad, las cuestionables gestiones de vida y muerte, los conflictos y litigios entre personas y la fragilización de las tramas sociales.

\section{Trabajar en contextos castigados}

La acumulación de décadas de investigaciones, políticas e intervenciones diagnósticas y preventivas en salud pública exigen una revisión profunda del trabajo antropológico, de su metodología y viabilidad bajo los regímenes de confinamiento que, en sus diferentes variantes, tienen la criminalización como una de sus bases. Es necesario considerar que la viabilidad, fiabilidad y potenciales efectos deletéreos se extreman cuando el objeto del análisis es la acusación, la sanción moral y la denuncia judicial y sus derivados, como es el caso de las denuncias persona a persona en contextos de proximidad.

En el trabajo antropológico sobre malestares y afecciones de salud, los modos de abordaje, los vínculos con las personas afectadas, los modos de análisis y registro de la información son sometidos a revisión y análisis exhaustivo respecto de la calidad y fiabilidad de la información producida. Asimismo, dichos trabajos son revisados para evaluar los efectos y consecuencias posibles para aquellas personas con las que se trabaja de forma colaborativa en contextos sociales específicos. Como innumerables estudios han señalado (Bourgois 2002; Bourgois, Lettiere y Quesada 1997; Epele 2008b; Koester 1994; Singer 1998), cuando se trabaja con personas y grupos afectados por problemas de salud eclipsados por cuestiones de normatividad extrema, acusación social, sanción moral, penalización y criminalización, se deben contemplar ciertas cuestiones metodológicas y técnicas para evitar que el trabajo antropológico reproduzca las expectativas sociales y normativas y profundice las consecuencias de estos procesos en las personas y conjuntos que los padecen.

Por un lado, en ciertas aflicciones y conjuntos sociales afectados por las mismas se sedimentan, intensifican y expresan, a veces con crueldad, ciertas normatividades referentes al dominio de experiencias en las que emergen las dolencias. Si bien este es un problema inherente a toda experiencia social, en zonas de intensa normatividad confluyen diferentes disposiciones (expertas, religiosas, legas, híbridas, etc.) que afectan y son apropiadas por diversos conjuntos sociales bajo el formato del 
imperativo universal, es decir, sin contemplar las condiciones materiales de vida, las tradiciones locales y las políticas de enunciación en sectores segregados. Cuando se abordan desde la antropología y las ciencias sociales, los modos de narrar las experiencias y aflicciones tienden mayormente a ajustarse a las respuestas que las propias personas consultadas consideran como "adecuadas", "normales" y "saludables" guiadas por los profesionales que las realizan y los discursos dominantes. Por otro lado, ciertas aflicciones se convierten en objeto de discursos públicos y de metáforas cargadas de sanciones sociales y acusaciones morales, en ocasiones contradictorios entre sí. En estos casos, las personas afectadas se convierten en el blanco de procesos colectivos de culpabilización y responsabilización. Por tanto, estas personas suelen verse empujadas a desarrollar acciones y producciones simbólicas para evitar ser incluidas en las categorías estigmatizadas. Además, hay otras afecciones de salud que incluyen ciertos comportamientos que han sido ilegalizados penalmente. La aproximación antropológica no solo debe contemplar los efectos de la penalización (no revelación de información, ocultamientos, etc.), sino también diseñar estrategias de documentación y análisis que impidan exponer a los sujetos a la autoincriminación respecto de aquellas prácticas criminalizadas que son exploradas.

Las etnografías in situ, es decir, en los mismos contextos sociales en los que ciertas afecciones tienen lugar y las personas afectadas viven, han tenido un rol central para poner en evidencia los alcances de estos procesos (Bourgois, Lettiere y Quesada 1997; Epele 2010). A diferencias de otros métodos cualitativos, la inmersión y la observación-participante se han convertido en un enfoque estratégico. No solo permiten documentar las prácticas y perspectivas de los propios actores sociales a escala de sus condiciones materiales y sociales de vida, sino que también muestran las dificultades teórico-metodológicas que incluyen otros enfoques cuali-cuantitativos (Epele 2010). Con la etnografía se ha podido documentar que las encuestas y entrevistas incluyen amplios e inciertos márgenes de error en la investigación de estas temáticas. Dichas herramientas producen como efecto secundario una profundización de las distancias entre las expectativas de los investigadores y las experiencias propias de los sujetos que se está investigando. Las distorsiones de magnitud desconocida no se pueden entender en términos de lógicas de verdad-mentira, es decir, de engaños calculados o instrumentalidad en la enunciación. Esta mirada vuelve a situar el problema del lado de la gente con la que se trabaja. Es una mirada que busca eludir la problemática de la artificialidad de los instrumentos y su mimetización con las intervenciones por parte de representantes del Estado, profesionales de salud y de las fuerzas de seguridad. Esta abrupta caída de los poderes de resolución de entrevistas y encuestas no es algo que puede "arreglarse" fácilmente con alguna sofisticación en las preguntas, con modificaciones en las secuencias de indagación o en los recursos retóricos de enunciación.

Además del problema de la confiabilidad de la información en el estudio e intervención de problemas de salud que están sujetos a la criminalización y penalización, deben considerarse las particularidades de los dispositivos legales-jurídicos 
que atraviesan los contextos histórico-políticos específicos. Específicamente, los medios por los que circula la información deben ser lo más confiables posibles, es decir, por fuera de los circuitos de vigilancia y de ciber-patrullaje a las que ciertas tecnologías y redes sociales están expuestas.

Para que los abordajes se adecuen a estas temáticas, se hace ineludible repensar el trabajo antropológico. No solo se debe comprender como un ejercicio colaborativo con la gente, sino que también se debe entender como un quehacer que implica tiempo. En estos estudios el tiempo es necesario para, como investigadoras, poder ser afectadas por los modos de vida de las personas y encontrar con los mismos actores sociales las maneras en que traducen sus experiencias a diferentes públicos para evitar dejarlos expuestos, sujetos a sanciones y criminalizaciones. Los sujetos individuales y colectivos tienen perspectivas y experiencias previas con gente que hace preguntas. Esto puede incluir investigaciones que han venido de la mano de otras agendas y objetivos. En el trabajo en zonas de marginación, las condiciones de extrañamiento se invierten. Las antropólogas entran a esa categoría de "aquellas personas" que pueden dejarlos expuestos a la mirada y sanciones de otros.

\section{Consideraciones finales}

Y así, seguimos andando, curtidos de soledad.

$\mathrm{Y}$ en nosotros nuestros muertos pa' que nadie quede atrás.

Yo tengo tantos hermanos que no los puedo contar.

Atahualpa Yupanki (Los hermanos, 1953)

Teniendo como meta la delimitación del problema referido a acusaciones, sanciones y denuncias de persona a persona bajo el régimen de confinamiento, este trabajo interroga y problematiza ciertas cuestiones preliminares necesarias para su abordaje. La importancia de implementar disposiciones tempranas de prevención, cuidado y asistencia respecto de la pandemia es indiscutible. De igual forma, es vital la necesidad de facilitar los recursos materiales para la supervivencia inmediata de los conjuntos sociales más afectados por la profundización de las crisis económicas. Sin embargo, es igual de importante y urgente considerar la materia de la que están hechas las disposiciones, las decisiones sobre a quiénes se delega el poder de hacerlas cumplir y sancionar sus infracciones y la sensibilidad que se tiene respecto a contextos y poblaciones específicas. A la misma escala de importancia, se hace ineludible considerar qué tipos de tensiones (contradicciones, interferencias) tienen estas normativas con las campañas sanitarias relativas al cuidado, los dispositivos ampliados de asistencia, la publicación de estadísticas de contagios y muertes. De igual forma es indispensable analizar las especificidades y adecuaciones que tiene cada contexto particular. 
Este problema no es algo nuevo. Desde la constitución misma de la antropología de la salud, este problema se ha actualizado de diversas formas, con diferentes rostros, regiones, contextos y coyunturas histórico-política-económicas. La importancia de considerar en detalle la naturaleza de las intervenciones no solo es relevante en catástrofes de salud, sino también en dolencias en apariencia menores y que afectan a personas particulares. Como ciertos trabajos clásicos señalan, cuando el tratamiento a algunas dolencias fuerza a las personas a interrumpir sus rutinas cotidianas, les producen sufrimientos, convierten sus cuerpos en objeto de diferentes saberes y prácticas y se instala la incertidumbre sobre su evolución y muerte posible, inevitablemente irrumpen ciertos interrogantes sobre los sentidos de sus vidas. Generalmente, las personas se preguntan sobre el porqué de las enfermedades. Como parte de estas interrogaciones, se revisan las prioridades y justificaciones de sus modos de vivir, antes alineadas y alienadas por los destinos que las agendas político-económicas han envasado y vendido en trayectorias de vida (Kleinman1991; Taussig 1992; Young 1997). De acuerdo a Taussig (1992), esta particular ruptura sentida al resquebrar los cimientos de las vidas convierte a las personas en sujetos más maleables y dependientes. Este resquebrajamiento las deja más expuestas a que se les "hagan carne" de diversas doctrinas y prácticas epistemológicas y ontológicas, en particular de las biomédicas y del capitalismo. Además, y siguiendo a Scarry (1985), las intervenciones directas y crueles sobre los cuerpos, son consideradas como antiguas tácticas para hacer real y ganar facticidad, reconocimiento y legitimidad por parte de regímenes de poder ilegítimos o de frágil institucionalidad.

Es evidente que ciertos aspectos de estos argumentos han perdido pertinencia y eficacia con los años. Específicamente, son ya antiguas las formas que en estos argumentos asumen las disputas entre la antropología y la biomedicina. Dichas tensiones estaban planteadas en un escenario previo a la proletarización de los profesionales de la salud, el protagonismo central de las industrias farmacéuticas, la radical desigualdad en el acceso a sistemas eficientes de cuidado y salud y la generalización de democracias en la región. Estas perspectivas, sin embargo, siguen teniendo relevancia y arrojando luz sobre las experiencias de sufrimiento y de enfermedad, específicamente, en contextos catastróficos como los actuales. Bajo estas condiciones, el miedo, desasosiego, angustia e incertidumbre se convierten en tierra fértil tanto para el surgimiento de formas de vivir de otro modo, orientadas al bienestar y al bien común, como para la proliferación táctica de lógicas de poder que las sofocan en su emergencia misma, desdibujan su potencial y las encarrilan nuevamente hacia aquellas formaciones que las lógicas de poder usufructúan, explotan y saben gobernar.

Volviendo al problema de las acusaciones y denuncias en contextos de proximidad, el examen de las cuestiones que lo atraviesan en la microescala del aislamiento no solo expresa las rápidas y profundas reorientaciones de los vínculos entre salud pública, sanciones, legislación y fuerzas de seguridad. Este examen también abre la interrogación, desde un lugar ex-céntrico a los binarismos oposicionales 
acerca de los modos en que estas prácticas traducen y reproducen lógicas acusatorias entre facciones radicalizadas que contaminan la sociedad a macroescala. Dichas lógicas acusatorias comprometen el bienestar, supervivencia y la misma posibilidad de sociabilidad presente y futura. Por último, y lo que es más importante, se hace ineludible para avanzar e innovar "volver al futuro" (Quijano 2014). Se hace necesario situar los difíciles desafíos y gestionar los inéditos problemas de proporciones catastróficas que la pandemia y los confinamientos han provocado, a la luz de los horizontes que las realidades y tradiciones de salud y de cuidado de la región proyectan, los pensares-sentires que las han forjado (Escobar 2016), y la historia de emergencias sociales que las han modelado.

\section{Referencias}

1. Abadía-Barrero, César. 2004. "Políticas y sujetos de sida en Brasil y Colombia". Revista Colombiana de Antropología 40: 123-154. https://doi.org/10.22380/2539472X.1217

2. Aljanati, Lucía; Joice Barbosa Becerra, José María Bompadre, Avelina Brown, Natalia Castelnuovo Biraben, Sasha Camila Cherñavsky, Julia Colla, Juan Engelman, Carla Golé, Ana Cecilia Gerrard, Valeria Herrera, Carolina Maidana, Rocío M. Míguez Palacio, Eugenia Morey, Luciana Quispe, Juan Carlos Radovich, Paula Reinoso, Sofía Rodríguez Ramírez, Mariana Schmidt, Liliana Tamagno, Sandra Tolosa, Sebastián Valverde y Sofía Varisco 2020. Pueblos indígenas y Covid-19. Principales lineamientos de un informe colaborativo. Buenos Aires: Editorial de la Facultad de Filosofía y Letras (UBA); Clacso.

3. Angulo, Martín. 2020. "Violación de la cuarentena: seis casos en donde los hechos desafían a las leyes". Infobae, 9 de junio. https://www.infobae.com/sociedad/2020/06/09/ violacion-de-cuarentena-seis-casos-en-donde-los-hechos-desafian-a-las-leyes/

4. Auyero, Javier y Débora Swistum. 2008. Inflamable. Estudio del sufrimiento ambiental. Buenos Aires: Paidós.

5. Basile, Gonzalo. 2020. "SARS-CoV-2 en América Latina y Caribe: las tres encrucijadas para el pensamiento crítico en salud”. Ciencia \& Saúde Colectiva 25 (9): 3557-3562. https://doi.org/10.1590/1413-81232020259.20952020

6. Betancourt, Marcos. 2020. "Para frenar la violencia policial durante el coronavirus". Página 12, 2 de abril. https://www.pagina12.com.ar/256860-para-frenar-la-violenciapolicial-durante-el-coronavirus

7. Biehl, Joao. 2013. “The Judicialization of Biopolitics: Claiming the Right to Pharmaceuticals in Brazilian Courts". American Ethnologist 40 (3): 419-436. https://doi.org/10.1111/amet.12030

8. Biehl, Joao. 2007. Will to Live. AIDS Therapies and the Politics of Survival. Nueva Jersey: Princeton University Press.

9. Bourgois, Philippe. 2002. "Anthropology and Epidemiology on Drugs: The Challenges of Cross-Methodological and Theoretical Dialogue". International Journal of Drug Policy 13 (4): 249-259. https://doi.org/10.1016/S0955-3959(02)00115-9 
10. Bourgois, Philippe, Mark Lettiere y James Quesada. 1997. "Social Misery and the Sanctions of Substance Abuse: Confronting HIV Risk among Homeless Heroin Addicts in San Francisco”. Social Problems 44 (2): 155-173. https://doi.org/10.2307/3096940

11. Biernat, Carolina. 2018. "Cuando los enfermos van a la justicia. Denuncia de varones por contagio venéreo y prácticas punitivas en la provincia de Buenos Aires (1936-1954)”. Revista Historia y Justicia 10: 104-129. https://doi.org/10.4000/rhj.1295

12. Briggs, Charles y Clara Mantini-Briggs. 2003. Stories in the Time of Cholera. Racial Profiling During a Medical Nightmare. Berkeley: University of California Press.

13. Castro, Arachu y Paul Farmer. 2003. "El sida y la violencia estructural. La culpabilización de la víctima”. Cuadernos de Antropología Social 17: 29-47. http://www.redalyc.org/ articulo.oa?id=180913909003

14. CELS. 2016. El impacto de las politicas de drogas en los derechos humanos. Buenos Aires: Centro de Estudios Legales y Sociales.

15. Caram, Sofía. 2020. "Línea 134: cómo se hacen las denuncias a quienes no cumplen el aislamiento por coronavirus". Página 12, 20 de marzo. https://www.pagina12.com.ar/ 254023-linea-134-como-se-hacen-las-denuncias-a-quienes-no-cumplen-e

16. Comaroff, Jean y John Comaroff, eds. 2006. Law and Disorder in the Postcolony. Chicago: The University of Chicago Press.

17. Crawford, Robert. 1994. "The Boundaries of the Self and the Unhealthy Other: Reflection on Health, Culture and AIDS”. Social Science \& Medicine 38 (10): 1347-1365. https://doi.org/ 10.1016/0277-9536(94)90273-9

18. Cueto, Marcos. 2009. "Cólera y dengue al final del siglo XX y comienzos del siglo XXI: salud y la cultura de la supervivencia”. En Historia social urbana. Espacios y flujos, editado por Eduardo Kingman Garcés, 253-273. Quito: Flacso. https://biblio.flacsoandes.edu.ec/ catalog/resGet.php? resId=25597

19. Epele, María. 2010. Sujetar por la herida. Buenos Aires: Paidós.

20. Epele, María E. 2008b. "Privatizando el cuidado: desigualdad, intimidad y uso de drogas en el gran Buenos Aires, Argentina". Antípoda. Revista de Antropología y Arqueología 6: 293-312. https://doi.org/10.7440/antipoda6.2008.14

21. Epele, María. 2007. "La lógica de la sospecha. Sobre la criminalización del uso de drogas, complots y barreras de acceso al sistema de la salud”. Cuadernos de Antropología Social 25: 151-168. http://revistascientificas.filo.uba.ar/index.php/CAS/article/view/4383/3887

22. Escobar, Arturo. 2016. "Sentipensar con la tierra: las luchas territoriales y la dimensión ontológica de las epistemologías del sur”. Revista Iberamericana de Antropología (AIBR) 11 (1): 11-32. https://doi.org/10.11156/aibr.110102

23. Farmer, Paul. 2003. Pathologies of Power. Health, Human Rights and the New War on the Poor. Berkeley: University of California Press.

24. Farmer, Paul. 1999. Infections and Inequalities. The Modern Plagues. Berkeley: University of California Press.

25. Farmer Paul. 1992. Aids \& Accusation. Haiti and the Geography of the Blame. Berkeley: University of California Press. 
Antipod. Rev. Antropol. Arqueol. n. ${ }^{\circ} 44 \cdot$ Bogotá, julio-septiembre $2021 \cdot$ ISSN 1900-5407 · e-ISSN 2011-4273 · pp. 119-144 https://doi.org/10.7440/antipoda44.2021.06

26. Fassin, Didier. 2007. When Bodies Remember. Experiences and Politics of AIDS in Africa. Berkeley: University of California Press.

27. Foucault, Michel. 2006. Seguridad, territorio y población. Cursos de Collège de France (1977-1978). Buenos Aires: Fondo de Cultura Económica.

28. Foucault, Michel. 2005. El poder psiquiátrico. Cursos de Collège de France (1973-1974). Buenos Aires: Fondo de Cultura Económica.

29. Gargarella, Roberto. 2020. "Los riesgos del estado de emergencia en América Latina”, La Nación, 13 de abril. https://www.lanacion.com.ar/

30. Goldberg, Alejandro. 2013. "Un abordaje comparativo en torno a la incidencia de la tuberculosis en migrantes bolivianos en Buenos Aires y São Paulo". Revista Interdisciplinar da Mobilidade Humana 40: 93-106. https://www.scielo.br/j/remhu/a/5bh4M9bwPfSGQ7 rm4PnfSnq/?lang=es\&format=pdf

31. González Díaz, Marcos. 2020. "Coronavirus. El preocupante aumento de agresiones en México contra personal médico que combate el Covid-19”, BBC News, 17 de abril. https:// www.bbc.com/mundo/noticias-america-latina-52319044

32. Gonzalo, Ángel. 2020. "Cuando la denuncia lleva tu nombre”, Amnesty International, 9 de octubre. https://www.es.amnesty.org/

33. Grimberg, Mabel. 2005. "Estigmatización y discriminación social relacionada al VIH en países de América Latina: sexualidad y uso de drogas en jóvenes en contextos de precarización social urbana". En VIH/SIDA, estigma y discriminación: un enfoque antropológico. División de políticas culturales y diálogo intercultural 20: 11-22. París: Unesco. https://www.conicet.gov.ar/new_scp/detalle.php?keywords=\&id=19487\&articulos =yes\&detalles=yes\&art_id=1457641

34. Kleinman, Arthur y Joan Kleinman. 1991. "Suffering and its Professional Transformation: Toward an Ethnography of Interpersonal Experience". Culture Medicine and Psychiatry 15: 275-301. https://doi.org/10.1007/BF00046540

35. Koester, Stephen. 1994. "Copping, Running, and Paraphernalia Laws: Contextual Variables and Needle Risk Behavior among Injection Drug Users in Denver". Human Organization 53 (3): 287-295. https://doi.org/10.17730/humo.53.3.g86872408528021h

36. Horton, Richard. 2020. "Covid-19 is Not a Pandemic". The Lancet 396 (10255): 874. https://doi.org/10.1016/S0140-6736(20)32000-6

37. Inchaurraga, Silvia, comp. 2003. Las drogas: entre el fracaso y los daños de la prohibición. Nuevas perspectivas en el debate despenalización-legalización. Rosario: CEAD-UNR/ARDA.

38. Iriart Celia, Howard Waitzkin, Jaime Breilh, Alfredo Estrada y Emerson Elías Merhy. 2002. "Medicina social latinoamericana: aportes y desafíos". Revista Panamericana de Salud Pública 12 (2): 128-136. https://www.scielosp.org/article/ssm/content/raw/?resource_ ssm_path=/media/assets/rpsp/v12n2/11619.pdf

39. Lavieri, Omar. 2020. "El juzgado a cargo de los casos de violación de la cuarentena estableció un protocolo para actuar en la emergencia: hubo más de 8.500 imputados", Infobae, 1 de abril. https://www.infobae.com/sociedad/policiales/2020/04/01/ el-juzgado-a-cargo-de-los-casos-de-violacion-de-la-cuarentena-establecio-un-protocolopara-actuar-en-la-emergencia-hubo-mas-de-8500-imputados/ 
40. Mannino, Pablo. 2015. "Controvertido juicio en Mendoza por contagio de VIH-SIDA”, La Nación, 30 de octubre. https://www.lanacion.com.ar/sociedad/controvertido-juicio-enmendoza-por-contagio-de-vih-nid1841064/

41. Margulies, Susana. 2014. La atención médica del VIH-SIDA. Un estudio de la antropología de la medicina. Buenos Aires: Editorial de la Facultad de Filosofía y letras, Universidad de Buenos Aires.

42. Menéndez, Eduardo L. 2009. "De racismos, esterilizaciones y algunos otros olvidos de la antropología y epidemiología mexicanas". Salud Colectiva 5 (2): 155-179. https://www. scielosp.org/pdf/scol/2009.v5n2/155-179/es

43. Meyer, Adriana. 2020. "Brutal ataque policial, con torturas y ataque sexual a cuatro jóvenes quom en Chaco", Página 12, 3 de junio. https://www.pagina12.com. ar/269912-brutal-ataque-policial-con-torturas-y-abuso-sexual-a-cuatro-

44. Ministerio de Salud de Argentina. 2020. Información epidemiológica. https://www. argentina.gob.ar/salud/coronavirus/informacion-epidemiologica

45. Molina Serra, Ainhoa. 2018. "Esterilizaciones en Perú: control reproductivo desde el poder y las familias indígenas". Tesis doctoral, Departamento de Antropología Social y Cultural, Universidad Autónoma de Barcelona.

46. Nino, Carlos. 2005. Un país al margen de la ley. Buenos Aires: Emecé.

47. O’ Donnell, Guillermo. 2002. "Las poliarquías y la (in)efectividad de la ley en América Latina”. En La (in)efectividad de la ley y la exclusión en América Latina, editado por Juan E. Méndez, Guillermo O’Donnell y Paulo Sérgio Pinheiro, 305-333. Buenos Aires: Paidós.

48. Onusida. 2008. Penalización de la transmisión del VIH. Ginebra: Programa Conjunto de las Naciones Unidas sobre el VIH-SIDA. https://www.unaids.org/sites/default/files/ media_asset/jc1601_policy_brief_criminalization_long_es.pdf

49. Parker, Richard. 2009. "Unintended Consequences: Evaluating the Impact of HIV and AIDS on Sexuality Research and Policy Debates". Cadernos de Saúde Pública 25 (2): S251-S258. http://dx.doi.org/10.1590/S0102-311X2009001400007

50. Parker, Richard, Delia Easton y Charles Klein. 2000. "Structural Barriers and Facilitators in HIV Prevention: A Review of International Research". AIDS 14 (1): 17-46. https://doi. org/10.1007/0-306-47518-9_2

51. Pascual, Ignacio y Gasparini Juan. 2020. “Covid-19: Ley penal en blanco y principio de legalidad”, Sistema Argentino de Información Jurídica, 27 de mayo. http://perso.unifr.ch/ derechopenal/assets/files/articulos/a_20200708_01.pdf

52. Pecheny, Mario. 2001. La construction de l'avortement et du sida en tant que questions politiques: le cas de l'Argentine. Lille: ADT, Presses Universitaires du Septentrion.

53. Petryna, Adriana. 2002. Life Exposed. Biological Citizen after Chernobyl. Princeton: Princeton University Press.

54. Quijano, Anibal. 2014. "El regreso del futuro y las cuestiones del conocimiento". En Cuestiones y horizontes. Antología Esencial, editado por Danilo Clímaco, 833-847 Buenos Aires: Clacso.

55. Scarry, Elaine. 1985. The Body in Pain. The Making and the Unmaking the World. Nueva York: Oxford University Press. 
Antipod. Rev. Antropol. Arqueol. n. ${ }^{\circ} 44 \cdot$ Bogotá, julio-septiembre $2021 \cdot$ ISSN 1900-5407 · e-ISSN 2011-4273 · pp. 119-144 https://doi.org/10.7440/antipoda44.2021.06

56. Singer, Merril. 2008. Introduction to Syndemics. A Critical Systems Approach to Public and Community Health. San Francisco: Wiley Imprint.

57. Singer, Merril.1998. The Political Economy of AIDS. Nueva York: Baywood Publishing.

58. Svampa, Maristella y Enrique Viale. 2014. Mal desarrollo. La Argentina del extractivismo y despojo. Buenos Aires: Katz Editores.

59. Taussig, Michel. 1992. "La reificación y la conciencia del paciente". En Un gigante en convulsiones. El mundo humano como sistema nervioso en emergencia permanente, 110-143. Barcelona: Gedisa.

60. Tiscornia, Sofía, comp. 2004. Estudios de antropología jurídica. Buenos Aires: Editorial Antropofagia; FFyL.

61. Tiscornia, Sofía, Roberto Kant de Lima y Lucía Eilbaum, comps. 2009. Burocracias penales, administraciones institucionales de conflictos y ciudadanía. Experiencias comparadas entre Argentina y Brasil. Buenos Aires: Editorial Antropofagia.

62. Tarducci, Mónica y Débora Daich. 2018. “Antropólogas feministas por el derecho a decidir. Aportes para una lucha para la despenalización y legalización del aborto en Argentina”. Publicar 24: 8-26. https://ppct.caicyt.gov.ar/index.php/publicar/article/ viewFile/ 14144/45454575758950

63. Unaids. 2013. Judging the Epidemic. A Judicial Handbook on HIV, Human Rights and the Law. Ginebra: United Nations Programme on HIV-AIDS. https://www.unaids.org/sites/ default/files/media_asset/201305_Judging-epidemic_en_0.pdf

64. Ventura Santos, Ricardo, Ana Lucía Pontes y Carlos E. A. Coimbra Jr. 2020. "Un 'hecho social total': Covid-19 y pueblos indígenas en Brasil”. Cadernos de Saúde Pública 36 (10): 1-4. https://doi.org/10.1590/0102-311X00268220

65. Zunino, Daiana. 2020. “Coronavirus en la Argentina. Habrá multas de hasta \$17.000 para quienes discriminen a los trabajadores de salud en la ciudad”, La Nación, 28 de mayo. https://www.lanacion.com.ar/sociedad/por-pandemia-multaran-quienes-discriminentrabajadores-esenciales-nid2370715/ 